\title{
Eighth World Conference on Lung Cancer, Dublin, August 1997
}

\author{
R J Fergusson
}

The prognosis of patients with lung cancer remains bleak, with most presenting with advanced unresectable disease. In recent years much effort has been put into attempts to improve survival with systemic chemotherapy, and newer agents are appearing. The results of published studies of drug treatment have been greeted with enthusiasm by oncologists and nihilism by many chest physicians. ${ }^{1} \mathrm{~A}$ meta-analysis published in the British Medical fournal has shown survival benefit with the addition of platinum based chemotherapy to all other treatment regimes (surgery, radiotherapy, and best supportive care). Chemotherapy has therefore been thrust into the limelight ${ }^{3}$ and was at the forefront of a recent international lung cancer meeting.

The 8th World Conference on Lung Cancer was held in Dublin under the auspices of the International Association for the Study of Lung Cancer (IASLC) in August 1997. The IASLC has been in existence for 23 years and holds such a Congress every three years. More than 2500 delegates attended the scientific sessions over the five days of the meeting which addressed all the important recent advances in the prevention, epidemiology, diagnosis, and management of this disease. Abstracts from the meeting have been published in Lung Cancer, the IASLC's own journal. ${ }^{45}$

\section{Chemotherapy}

Results in non-small cell lung cancer (NSCLC) in line with the meta-analysis ${ }^{2}$ were presented from two large randomised studies by Cullen in the UK. In the first, standard chemotherapy (mitomycin, ifosfamide and cisplatinum (MIC)) prior to conventional radical radiotherapy was compared with radiotherapy alone in 447 patients with NSCLC; $52 \%$ of patients in the chemotherapy arm showed a response to this treatment and there was a trend to increased survival in this arm (median survival 13 months versus 9.9 months, $24 \%$ alive versus $18 \%$ at two years and $10 \%$ versus $4 \%$ at four years). These data did not reach statistical significance. In the second study the same MIC regime was added to best supportive care and compared with this alone in 350 patients with extensive NSCLC. Response rates in drug treated patients were lower than in the first study but a significant survival advantage (median survival 6.9 versus 4.8 months, $28 \%$ versus $18 \%$ alive at one year and $6 \%$ versus $4 \%$ at two years) was seen in patients receiving chemotherapy. Although these differences would appear to be small, they also showed that, in a cohort of 189 patients where data were available, there was an improved quality of life in patients treated with chemotherapy. The cost effectiveness of chemotherapy in NSCLC was questioned by other workers but data from Canada suggested that, even in patients with advanced disease, this treatment could be justified on financial grounds alone. The treatment of lung cancer in the elderly remains controversial ${ }^{67}$ but data presented at the Congress showed that it was not an adverse predictive factor for chemotherapy in either small cell lung cancer (SCLC) or NSCLC.

Clearly, newer more effective agents are required and data showing reasonable activity with compounds such as vinorelbine, the taxols, camptothecins, and gemcitabine were all presented. In a multicentre British trial gemcitabine was compared with best supportive care in 299 patients with NSCLC. The drug was well tolerated and less palliative radiotherapy was required in the gemcitabine treated patients $(7.3 \%$ compared with $42 \%)$. Two randomised phase II studies showed that gemcitabine was at least as effective as standard cisplatinum plus ifosfamide chemotherapy but much less toxic for the patient. It would seem important that any new agent in this disease is compared with "standard" chemotherapy and that quality of life rather than just response and survival are measured.

These new agents have also been tried in patients with SCLC. Many of the studies were phase II in design and in some pretreated patients were included. The importance of discovering newer agents was highlighted by a study that showed no change in the results of chemotherapy over the past two decades from a single centre with a database of more than 2000 patients with extensive stage SCLC undergoing chemotherapy with a number of different regimes.

\section{Radiotherapy}

The final results of the CHART (continuous hyperfractionated accelerated radiotherapy) trial were presented. Five hundred and sixty three patients with NSCLC were randomised in 13 cooperating centres to receive either CHART $(1.5 \mathrm{~Gy} /$ fraction three times per day for 12 consecutive days) or conventional radiotherapy (60 Gy in 30 fractions). Survival was the main end point of the study and in the CHART group there was an absolute improvement in survival at two years of $9 \%$ (29\% versus $20 \%)$ with even better results seen in the subgroup with squamous cell histology (33\% versus 19\% two year survival). These survival differences between treatments have continued up to four years. CHART treatment was associated with more severe oesophagitis but there were no long term sequelae. It remains to be seen whether this form of radical radiotherapy becomes "standard". There are obvious resource implications for treating patients so intensively. Two papers suggested that it might be possible to avoid weekend therapy without compromising results.

\section{Other areas}

The role of minimal invasive techniques using video assisted thoracic surgery (VATS) was discussed. Impressive 
results were presented in stage I disease but there are few randomised studies comparing VATS with standard thoracotomy. More controversial papers were presented concerning the manipulation of cigarette production to cause and enhance addiction, the question of gene therapy for lung cancer, and the possibility of "chemoprevention" of at risk patients with various compounds. The final results of these studies are eagerly awaited.

The patterns of managing bronchial carcinoma by chest physicians in the United Kingdom are variable ${ }^{78}$ and it was striking just how few British chest physicians were present at this major international meeting. Indeed, the IASLC has only 22 UK members, the majority of whom are oncologists. Lung cancer accounts for a large part of the workload of respiratory physicians who are responsible for making the diagnosis and initiating management plans. The international scientific community are pressing ahead with exciting new treatment regimes in this disease and it would seem vital for British thoracic medicine that we are involved in the design of future studies and participate in ongoing trials. ${ }^{1}$

Consultant Physician,

Eastern General Hospital,

R J FERGUSSON

Seafield Street,

Edinburgh EH6 7LN, UK

1 Spiro SG. Clinical trials in lung cancer: nihilism versus enthusiasm. Thorax 1997;52:598-604.

2 Non-Small Cell Lung Cancer Collaborative Group. Chemotherapy in nonsmall cell lung cancer: a meta-analysis using updated data in individual small cell lung cancer: a meta-analysis using updated data in individu
patients from 52 randomised clinical trials. BMF 1995;311:899-909.

patients from 52 randomised clinical trials. $B M F$ 1995;311:899-909.
3 Souhami RL. Chemotherapy in non-small cell lung cancer: time to reexamine our attitudes. Thorax 1996;51:231-2.

4 Abstracts 8th World Conference on Lung Cancer 10-15 August 1997 Dublin, Ireland. Lung Cancer 1997;18(Suppl 1):3-236.

5 Extended Abstracts 8th World Conference on Lung Cancer 10-15 August 1997, Dublin, Ireland. Lung Cancer 1997;18(Suppl 2):3-160.

6 Brown JS, Eraut D, Trask C, Davison AG. Age and the treatment of lung cancer. Thorax 1996;51:564-8.

7 Muers MF, Haward RA. Management of lung cancer. Thorax 1996;51: $557-60$.

8 Fergusson RJ, Gregor A, Dodds R, Kerr G. Management of lung cancer in south east Scotland. Thorax 1996;51:569-74. 


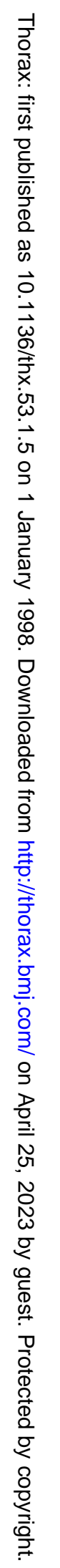

